\title{
THE IMPORTANCE OF STATISTICS IN HEALTH RESEARCH
}

David Coggon ${ }^{1}$

The enormous advances in medicine over the past two centuries have come about through the application of science to what previously had been a largely ineffective art, and statistical methods have been at the heart of this revolution. Their importance stems from the variability that is intrinsic to biological organisms and systems. If in the chemistry laboratory, we mix one chemical with another under standardised conditions, we expect always to obtain the same outcome. In contrast, only rarely do all humans or animals respond identically when exposed to the same health hazard or treatment.

Because of biological variability, the effects of factors that cause disease or determine its outcome can only be characterised meaningfully at group level. If a child with leukaemia is given specified chemotherapy, we cannot reliably predict whether or not the treatment will be successful in that individual, but if 1,000 such patients are treated, we may know with some confidence the proportion who will be cured.

Making sense of biological variability in groups of people or animals requires statistical methods. As a start, there is a need to summarise succinctly but meaningfully the distribution of relevant measures within groups. If a clinician presented a slide at a meeting, listing 200 individual measurements of birth weight from a group of babies, little information of value would be conveyed. Much more helpful would be to show summary measures of central tendency (e.g. mean or median) and dispersion (e.g. standard deviation, inter-quartile range), or to depict the distribution of birth weights graphically in a histogram. Techniques for summarising group data are known as "descriptive statistics".

Moving beyond this, there is often a need to extrapolate from observations in a sample of people to a wider population in whom the findings might usefully be applied. As a general rule, in the presence of unexplained variability, larger samples are less likely to be unrepresentative simply by chance, and the potential for chance error can be gauged by two methods of "statistical inference" - hypothesis testing and estimation with confidence intervals.

Statistical methods have underpinned many of the most important successes of modern medicine, preventing much morbidity and saving many lives. All health practitioners should therefore have at least a basic grasp of the relevant principles. They do not all need the mathematical skills to perform statistical calculations, but they should have sufficient understanding of descriptive statistics, p-values and confidence intervals to be able to interpret published results and apply them in their own practice.

1Professor of Occupational and Environmental Medicine - MRC Lifecourse Epidemiology Unit, University of Southampton, UK 